Proyecciones

Vol. 27, No 3, pp. 289-305, December 2008.

Universidad Católica del Norte

Antofagasta - Chile

\title{
POSITIVE MATRICES WITH PRESCRIBED SINGULAR VALUES *
}

\author{
EMEDIN MONTANO \\ UNIVERSIDAD DE MAGALLANES, CHILE \\ MARIO SALAS \\ UNIVERSIDAD CATÓLICA DEL NORTE, CHILE \\ and \\ RICARDO L. SOTO \\ UNIVERSIDAD CATÓLICA DEL NORTE, CHILE \\ Received : June 2008. Accepted : October 2008
}

\begin{abstract}
We consider the problem of constructing positive matrices with prescribed singular values. In particular, we show how to construct an $m \times n$ positive matrix, $m \geq n$, with prescribed singular values $\sigma_{1} \leq \sigma_{2} \leq \cdots \leq \sigma_{n}$
\end{abstract}

AMS classification : 15A18; 15A29.

Keywords : Singular values, positive matrices.

\footnotetext{
*Supported by Fondecyt 1085125 , Chile.
} 


\section{Introduction}

A singular value decomposition of a matrix $A \in \mathbf{C}^{m \times n}$ is a factorization $A=U \Sigma V^{*}$, where $\Sigma=\operatorname{diag}\left(\sigma_{1}, \sigma_{2}, \ldots, \sigma_{r}\right) \in \mathbf{R}^{m \times n}, r=\min \{m, n\}$, $\sigma_{1} \geq \sigma_{2} \geq \ldots \geq \sigma_{r} \geq 0$ and both $U \in \mathbf{C}^{m \times m}$ and $V \in \mathbf{C}^{n \times n}$ are unitary. The diagonal entries of $\Sigma$ are called the singular values of $A$. The columns $\mathbf{u}_{j}$ of $U$ are called left singular vectors of $A$ and the columns $\mathbf{v}_{j}$ of $V$ are called right singular vectors of $A$. Every $A \in \mathbf{C}^{m \times n}$ has a singular value decomposition $A=U \Sigma V^{*}$ and the following relations hold: $A \mathbf{v}_{j}=\sigma_{j} \mathbf{u}_{j}$, $A^{*} \mathbf{u}_{j}=\sigma_{j} \mathbf{v}_{j}$ and $\mathbf{u}_{j}^{*} A \mathbf{v}_{j}=\sigma_{j}$. If $A \in \mathbf{R}^{m \times n}$, then $U$ and $V$ may be taken to be real (see [3]).

In this paper we consider the following inverse singular value problem:

Problem: Given the real numbers $\sigma_{1} \geq \sigma_{2} \geq \cdots \geq \sigma_{n} \geq 0$, to construct an $m \times n, m \geq n$, entrywise positive matrix $A$ with singular values $\sigma_{i}$, $i=1, \ldots, n$.

In the case $m=n$, the problem has always a solution if $\sigma_{1}>\sigma_{2}$. We construct such a solution from the following two results, due to Fiedler [2], and related with the nonnegative inverse eigenvalue problem:

Lemma 1. [2] Let $\alpha>\beta \geq 0, \epsilon>0$. Then there exists a number $c>0$ such that the matrix

$$
\left(\begin{array}{ll}
\alpha & c \\
c & \beta
\end{array}\right) \text { has eigenvalues } \alpha+\epsilon, \beta-\epsilon .
$$

Lemma 2. [2] Let $A$ be a symmetric $m \times m$ matrix with eigenvalues $\alpha_{1}, \ldots, \alpha_{m}$, and let $\mathbf{u},\|\mathbf{u}\|=1$, be a unit eigenvector corresponding to $\alpha_{1}$. Let $B$ be a symmetric $n \times n$ matrix with eigenvalues $\beta_{1}, \ldots, \beta_{n}$, and let $\mathbf{v},\|\mathbf{v}\|=1$, be a unit eigenvector corresponding to $\beta_{1}$. Then for any $\rho$, the matrix

$$
S=\left(\begin{array}{cc}
A & \rho \mathbf{u v} \mathbf{v}^{T} \\
\rho \mathbf{v u} \mathbf{u}^{T} & B
\end{array}\right)
$$

has eigenvalues $\alpha_{2}, \ldots, \alpha_{m}, \beta_{2}, \ldots, \beta_{n}, \gamma_{1}, \gamma_{2}$, where $\gamma_{1}, \gamma_{2}$ are eigenvalues of the matrix

$$
\widehat{S}=\left(\begin{array}{cc}
\alpha_{1} & \rho \\
\rho & \beta_{1}
\end{array}\right) \text {. }
$$


To construct a positive matrix, no necessaryly symmetric, with prescribed singular values, we first use the below result, Lemma 4 , which shows how to construct an $n \times n$ nonnegative semibordered matrix

$$
C=\left(\begin{array}{cccc}
b_{1} & b_{2} & \cdots & b_{n} \\
& a_{2} & & \\
& & \ddots & \\
& & & a_{n}
\end{array}\right),
$$

with prescribed singular values and then, to obtain the required positive matrix, we apply a Brauer [1] type singular value perturbation result given in [4, Corollary 14], which shows how to modify a single singular value of an $n \times n$ matrix without changing any of the remaining singular values.

Lemma 3. The characteristic polynomial of the arrow matrix

is

$$
B=\left(\begin{array}{cccc}
b_{1} & b_{2} & \cdots & b_{n} \\
b_{2} & a_{2} & & \\
\vdots & & \ddots & \\
b_{n} & & & a_{n}
\end{array}\right)
$$

$$
p(\lambda)=\left(b_{1}-\lambda\right) \prod_{i=2}^{n}\left(a_{i}-\lambda\right)-\sum_{i=2}^{n} b_{i}^{2} \prod_{\substack{k=2 \\ k \neq i}}^{n}\left(a_{k}-\lambda\right) .
$$

Proof. By expanding

$$
\begin{aligned}
& p(\lambda)=\operatorname{det}(A-\lambda I) \\
& \left|\begin{array}{ccccc}
b_{1}-\lambda & b_{2} & b_{3} & \cdots & b_{n} \\
b_{2} & a_{2}-\lambda & & & \\
b_{3} & & a_{3}-\lambda & & \\
\vdots & & & \ddots & \\
b_{n} & & & & a_{n}-\lambda
\end{array}\right| \\
& =\left(b_{1}-\lambda\right) \prod_{i=2}^{n}\left(a_{i}-\lambda\right)-\sum_{i=2}^{n} b_{i}^{2} \prod_{\substack{k=2 \\
k \neq i}}^{n}\left(a_{k}-\lambda\right) \text {. }
\end{aligned}
$$


Lemma 4. Given the real numbers $\sigma_{1}>\sigma_{2} \geq \cdots \geq \sigma_{n}>0$, then there exists an $n \times n$ nonnegative matrix $C$ of the form (1.1) with singular values $\sigma_{1}, \sigma_{2}, \ldots, \sigma_{n}$.

Proof. Let $\mathrm{A}=\left(\begin{array}{ccccc}b_{1} & b_{2} & b_{3} & \cdots & b_{n} \\ & a_{2} & & & \\ & & a_{3} & & \\ & & & \ddots & \\ & & & & a_{n}\end{array}\right)$.

Then

$$
\begin{aligned}
& B=A A^{T}=\left(\begin{array}{lllll}
b_{1} & b_{2} & b_{3} & \cdots & b_{n} \\
& a_{2} & & & \\
& & a_{3} & & \\
& & & \ddots & \\
& & & & a_{n}
\end{array}\right)\left(\begin{array}{ccccc}
b_{1} & & & & \\
b_{2} & a_{2} & & & \\
b_{3} & & a_{3} & & \\
\vdots & & & \ddots & \\
b_{n} & & & & a_{n}
\end{array}\right) \\
& =\left(\begin{array}{ccccc}
\sum_{i=1}^{n} b_{i}^{2} & a_{2} b_{2} & a_{3} b_{3} & \cdots & a_{n} b_{n} \\
a_{2} b_{2} & a_{2}^{2} & & & \\
a_{3} b_{3} & & a_{3}^{2} & & \\
\vdots & & & \ddots & \\
a_{n} b_{n} & & & & a_{n}^{2}
\end{array}\right)
\end{aligned}
$$

is an $n \times n$ arrow symmetric matrix with characteristic polynomial

$$
p(\lambda)=\left(\sum_{i=1}^{n} b_{i}^{2}-\lambda\right) \prod_{i=2}^{n}\left(a_{i}^{2}-\lambda\right)-\sum_{i=2}^{n}\left(a_{i} b_{i}\right)^{2} \prod_{j=2 j \neq i}^{n}\left(a_{j}^{2}-\lambda\right)
$$

and eigenvalues $\sigma_{1}^{2}, \sigma_{2}^{2}, \ldots, \sigma_{n}^{2}$. We define

$$
a_{k}=\sqrt{\frac{\sigma_{k-1}^{2}+\sigma_{k}^{2}}{2}}, k=2,3, \ldots, n .
$$

Then, by taking $\lambda=\frac{\sigma_{k-1}^{2}+\sigma_{k}^{2}}{2}, k=2,3, \ldots n$, in (1.2), we have

$$
\prod_{i=2}^{n}\left(a_{i}^{2}-\frac{\sigma_{k-1}^{2}+\sigma_{k}^{2}}{2}\right)=0
$$


and

$$
-\left(a_{k} b_{k}\right)^{2} \prod_{\substack{j=2 \\ j \neq k}}^{n}\left(a_{j}^{2}-\frac{\sigma_{k-1}^{2}+\sigma_{k}^{2}}{2}\right)=\prod_{i=1}^{n}\left(\frac{\sigma_{k-1}^{2}+\sigma_{k}^{2}}{2}-\sigma_{i}^{2}\right) .
$$

Hence,

$$
b_{k}=\sqrt{-\frac{\prod_{i=1}^{n}\left(a_{k}^{2}-\sigma_{i}^{2}\right)}{a_{k}^{2} \prod_{\substack{i=2 \\ i \neq k}}^{n}\left(a_{i}^{2}-a_{k}^{2}\right)}}, k=2,3, \ldots, n .
$$

For $b_{1}$ we take $\lambda=\sigma_{1}^{2}$ in (1.2). Then

$$
\left(b_{1}^{2}+\sum_{i=2}^{n} b_{i}^{2}-\sigma_{1}^{2}\right) \prod_{i=2}^{n}\left(a_{i}^{2}-\sigma_{1}^{2}\right)-\sum_{i=2}^{n}\left(a_{i} b_{i}\right)^{2} \prod_{\substack{j=2 \\ j \neq i}}^{n}\left(a_{j}^{2}-\sigma_{1}^{2}\right)=p\left(\sigma_{1}^{2}\right)=0
$$

and

$$
\begin{aligned}
b_{1} & =\sqrt{\frac{1}{\prod_{i=2}^{n}\left(a_{i}^{2}-\sigma_{1}^{2}\right)}\left(\sum_{i=2}^{n} a_{i}^{2} b_{i}^{2} \prod_{\substack{j=2 \\
j \neq i}}^{n}\left(a_{j}^{2}-\sigma_{1}^{2}\right)+\sigma_{1}^{2} \prod_{i=2}^{n}\left(a_{i}^{2}-\sigma_{1}^{2}\right)\right)-\sum_{i=2}^{n} b_{i}^{2}} \\
= & \sqrt{\frac{\sum_{i=2}^{n} a_{i}^{2} b_{i}^{2} \prod_{\substack{j=2 \\
j \neq i}}^{n}\left(a_{j}^{2}-\sigma_{1}^{2}\right)}{\prod_{i=2}^{n}\left(a_{i}^{2}-\sigma_{1}^{2}\right)}+\sigma_{1}^{2}-\sum_{i=2}^{n} b_{i}^{2}} \\
= & \sqrt{\sum_{i=2}^{n} \frac{a_{i}^{2} b_{i}^{2}}{a_{i}^{2}-\sigma_{1}^{2}}-\sum_{i=2}^{n} b_{i}^{2}+\sigma_{1}^{2}}
\end{aligned}
$$

Thus,

$$
b_{1}=\sqrt{\left[\sum_{i=2}^{n}\left(\frac{b_{i}^{2}}{a_{i}^{2}-\sigma_{1}^{2}}\right)\right] \sigma_{1}^{2}+\sigma_{1}^{2}}
$$


Lemma 5. [4] Let $A$ be an $m \times n$ matrix with singular values $\sigma_{1} \geq \sigma_{2} \geq$ $\cdots \geq \sigma_{r} \geq 0, r=\min \{m, n\}$. Let $\mathbf{u}_{i} \mathbf{v}_{i}$, respectively, the left and right singular vectors corresponding to $\sigma_{i}, i=1, \ldots, r$. Let $\alpha \in \mathbf{R}$ such that $\alpha+\sigma_{i} \geq 0$. Then $A+\alpha \mathbf{u}_{i} \mathbf{v}_{i}^{T}$ has singular values

$$
\sigma_{1}, \ldots, \sigma_{i-1}, \sigma_{i}+\alpha, \sigma_{i+1}, \ldots, \sigma_{r}
$$

The paper is organized as follows: In section 2 we construct an $n \times n$ positive matrix with prescribed singular values by using results related with the symmetric nonnegative inverse eigenvalue problem. In section 3 we construct a positive matrix, not necessarily symmetric, from Lemmas 4 and 5 . In section 4 we consider the rectangular case $m \times n, m>n$. Finally, in section 5 we give some examples to illustrate the results.

\section{Positive matrices with prescribed singular values I}

In this section we construct a positive symmetric matrix with prescribed nonnegative eigenvalues, which in this case, are also its singular values.

Theorem 1. Given the real numbers $\sigma_{1}>\sigma_{2} \geq \cdots \geq \sigma_{n} \geq 0$, there exists a positive matrix $A$ with singular values $\sigma_{1}, \sigma_{2}, \ldots, \sigma_{n}$.

Proof. Let

$$
B_{k}=\left(\begin{array}{cc}
\frac{\sigma_{k}+\sigma_{n-k+1}}{2} & \frac{\sigma_{k}-\sigma_{n-k+1}}{2} \\
\frac{\sigma_{k}-\sigma_{n-k+1}}{2} & \frac{\sigma_{k}+\sigma_{n-k+1}}{2}
\end{array}\right), \quad k=1,2, \ldots, \frac{n}{2}, \text { even } n,
$$

with $B_{\frac{n+1}{2}}=\left(\sigma_{\frac{n+1}{2}}\right)$ for odd $n$. Thus, $B_{k}$ is a positive symmetric matrix with singular values $\sigma_{k}$ and $\sigma_{n-k+1}$ (eigenvalues $\sigma_{k}$ and $\sigma_{n-k+1}$ ). Then

$$
B=\left(\begin{array}{cccc}
B_{1} & 0 & \ddots & 0 \\
0 & B_{2} & \ddots & \ddots \\
\ddots & \ddots & \ddots & 0 \\
0 & \ddots & 0 & B_{\frac{n}{2}}
\end{array}\right),
$$

with $B_{\frac{n+1}{2}}$ in the last diagonal position if $n$ is odd, is an $n \times n$ nonnegative symmetric matrix with singular values $\sigma_{1}, \sigma_{2}, \ldots, \sigma_{n}$. In order to obtain a 
positive matrix with prescribed singular values let us consider $\mu_{1}>\mu_{2} \geq$ $\cdots \geq \mu_{n} \geq 0$, where

$$
\begin{aligned}
& \mu_{1}=\frac{\sigma_{1}+\sigma_{2}}{2} \\
& \mu_{i}=\sigma_{i}+\epsilon_{i} \leq \mu_{i-1}, \quad i=2, \ldots, \frac{n}{2}\left(\frac{n+1}{2} \text { for odd } n\right), \\
& \mu_{i}=\sigma_{i}, i=\frac{n+2}{2}, \ldots, n\left(\frac{n+3}{2} \text { for odd } n\right)
\end{aligned}
$$

with

$$
\epsilon=\frac{\sigma_{1}-\sigma_{2}}{2} \text { and } \epsilon_{i}=\frac{2 \epsilon}{n-2}=\frac{\sigma_{1}-\sigma_{2}}{n-2}
$$

Now we apply the Lemma $2, \frac{n-2}{2}$ times if $n$ is even $\left(\frac{n-1}{2}\right.$ times if $n$ is odd). In the first step of this process we apply Lemma 2 to the matrices

$$
B_{1}=\left(\begin{array}{ll}
\frac{\mu_{1}+\sigma_{n}}{2} & \frac{\mu_{1}-\sigma_{n}}{2} \\
\frac{\mu_{1}-\sigma_{n}}{2} & \frac{\mu_{1}+\sigma_{n}}{2}
\end{array}\right), \quad B_{2}=\left(\begin{array}{ll}
\frac{\mu_{2}+\sigma_{n-1}}{2} & \frac{\mu_{2}-\sigma_{n-1}}{2} \\
\frac{\mu_{2}-\sigma_{n-1}}{2} & \frac{\mu_{2}+\sigma_{n-1}}{2}
\end{array}\right),
$$

with singular values $\mu_{1}, \sigma_{n}$ and $\mu_{2}, \sigma_{n-1}$, respectively. From Lemma 1 , the matrix

$$
\left(\begin{array}{cc}
\mu_{1} & \rho \\
\rho & \mu_{2}
\end{array}\right) \text {, where } \rho=\left(\epsilon_{2}\left(\mu_{1}-\mu_{2}+\epsilon_{2}\right)\right)^{\frac{1}{2}},
$$

has eigenvalues $\mu_{1}+\epsilon_{2}$ and $\mu_{2}-\epsilon_{2}=\sigma_{2}$. The unitary vectors

$$
\mathbf{u}=\mathbf{v}=\left(\frac{1}{\sqrt{2}}, \frac{1}{\sqrt{2}}\right)^{T}
$$

are the Perron eigenvectors of $B_{1}$ and $B_{2}$, respectively. Then the $4 \times 4$ matrix

$$
\mathcal{B}_{1}=\left(\begin{array}{cc}
B_{1} & \rho \mathbf{u v}^{T} \\
\rho \mathbf{v} \mathbf{u}^{T} & B_{2}
\end{array}\right)
$$

is positive symmetric with singular values (eigenvalues) $\mu_{1}+\epsilon_{2}, \sigma_{2}, \sigma_{n-1}, \sigma_{n}$. In the second step of the process we again apply Lemma 2 to the matrices $\mathcal{B}_{1}$ and

$$
B_{3}=\left(\begin{array}{ll}
\frac{\mu_{3}+\sigma_{n-2}}{2} & \frac{\mu_{3}-\sigma_{n-2}}{2} \\
\frac{\mu_{3}-\sigma_{n-2}}{2} & \frac{\mu_{3}+\sigma_{n-2}}{2}
\end{array}\right)
$$


which has singular values $\mu_{3}$ and $\sigma_{n-2}$. The matrix

$$
\left(\begin{array}{cc}
\mu_{1}+\epsilon_{2} & \rho \\
\rho & \mu_{3}
\end{array}\right) \text {, where } \rho=\left(\epsilon_{3}\left(\mu_{1}+\epsilon_{2}-\mu_{3}+\epsilon_{3}\right)\right)^{\frac{1}{2}}
$$

has eigenvalues $\mu_{1}+\epsilon_{2}+\epsilon_{3}$ and $\mu_{3}-\epsilon_{3}=\sigma_{3}$. Now let $\mathcal{B}_{1} \mathbf{u}=\left(\mu_{1}+\epsilon_{2}\right) \mathbf{u}$, $\|\mathbf{u}\|=1$ and $B_{3} \mathbf{v}=\mu_{3} \mathbf{v}, \mathbf{v}=\left(\frac{1}{\sqrt{2}}, \frac{1}{\sqrt{2}}\right)^{T}$. Then the $6 \times 6$ matrix

$$
\mathcal{B}_{2}=\left(\begin{array}{cc}
\mathcal{B}_{1} & \rho \mathbf{u} \mathbf{v}^{T} \\
\rho \mathbf{v} \mathbf{u}^{T} & B_{3}
\end{array}\right)
$$

is positive symmetric with singular values $\mu_{1}+\epsilon_{2}+\epsilon_{3}, \sigma_{2}, \sigma_{3}, \sigma_{n-2}, \sigma_{n-1}, \sigma_{n}$. The process is continued until in the last step we apply Lemma 2 to the matrices $\mathcal{B}_{\frac{n-4}{2}}\left(\mathcal{B}_{\frac{n-3}{2}}\right.$ for odd $\left.n\right)$, of order $n-2(n-1$ for odd $n)$ and $B_{\frac{n}{2}}$ $\left(B_{\frac{n+1}{2}}\right.$ for odd $\left.n\right)$, which has the singular values $\mu_{\frac{n}{2}}$ and $\mu_{\frac{n+2}{2}}\left(\mu_{\frac{n+1}{2}}\right.$ for odd $n)$. The matrix

$$
\left(\begin{array}{cc}
\mu_{1}+\left(\epsilon-\epsilon_{\frac{n}{2}}\right) & \rho \\
\rho & \mu_{\frac{n}{2}}
\end{array}\right) \text {, where } \rho=\left(\epsilon_{\frac{n}{2}}\left(\mu_{1}+\left(\epsilon-\epsilon_{\frac{n}{2}}\right)-\mu_{\frac{n}{2}}+\epsilon_{\frac{n}{2}}\right)\right)^{\frac{1}{2}},
$$

has eigenvalues $\mu_{1}+\epsilon$ and $\mu_{\frac{n}{2}}-\epsilon_{\frac{n}{2}}=\sigma_{\frac{n}{2}}$. Then the $n \times n$ matrix

$$
A=\mathcal{B}_{\frac{n-2}{2}}=\left(\begin{array}{cc}
\mathcal{B}_{\frac{n-4}{2}} & \rho \mathbf{u v} \mathbf{v}^{T} \\
\rho \mathbf{v} \mathbf{u}^{T} & B_{\frac{n}{2}}
\end{array}\right) \text { for even } n
$$

or

$$
A=\mathcal{B}_{\frac{n-1}{2}}=\left(\begin{array}{cc}
\mathcal{B}_{\frac{n-4}{2}} & \rho \mathbf{u v} \mathbf{v}^{T} \\
\rho \mathbf{v} \mathbf{u}^{T} & B_{\frac{n+1}{2}}
\end{array}\right) \text { for odd } n,
$$

where $\mathbf{u}$ and $\mathbf{v}$ are the unitary eigenvectors of $\mathcal{B}_{\frac{n-2}{2}}\left(\mathcal{B}_{\frac{n-1}{2}}\right.$ for odd $\left.n\right)$ and $B_{\frac{n}{2}}\left(B_{\frac{n+1}{2}}\right.$ for odd $\left.n\right)$, respectively, is positive and since

$$
\mu_{1}+\epsilon_{2}+\cdots \epsilon_{\frac{n}{2}}=\mu_{1}+\epsilon=\sigma_{1},
$$

$A$ has the prescribed singular values $\sigma_{1}, \sigma_{2}, \ldots, \sigma_{n}$.

\section{Positive matrices with prescribed singular values II}

In this section we construct an $n \times n$ positive matrix $A$, no necessarily symmetric, with prescribed singular values $\sigma_{1}, \sigma_{2}, \ldots, \sigma_{n}$. First, by applying Lemma 4 we construct a nonnegative semibordered diagonal matrix $C$ of the form 


$$
C=\left(\begin{array}{ccccc}
b_{1} & b_{2} & b_{3} & \cdots & b_{n} \\
& a_{2} & & & \\
& & a_{3} & & \\
& & & \ddots & \\
& & & & a_{n}
\end{array}\right), b_{j}>0, j=1, \ldots, n .
$$

and then, by applying Lemma 5 , we add to the matrix $C$ the positive matrix $\alpha \mathbf{u v}^{T}$, where $\alpha>0$ and $\mathbf{u}$ and $\mathbf{v}$ are the left and right singular vectors of $C$ corresponding to its maximal singular value.

Theorem 2. Let the nonnegative real numbers $\sigma_{1}>\sigma_{2} \geq \cdots \geq \sigma_{n}>0$ be given. Then there exists an $n \times n$ positive matrix $A$ with singular values $\sigma_{1}, \sigma_{2}, \ldots, \sigma_{n}$

Proof. Let $\alpha>0$ such that $\tilde{\sigma}_{1}=\sigma_{1}-\alpha>\sigma_{2}$ and consider $\tilde{\sigma}_{1}>\sigma_{2} \geq$ $\cdots \geq \sigma_{n}$. Then, from Lemma 4 there exists an $n \times n$ nonnegative matrix $C$ of the form (3.1), with singular values $\widetilde{\sigma}_{1}, \sigma_{2}, \ldots, \sigma_{n}$. The arrow matrix $C C^{T}$ is nonnegative, irreducible, with Perron eigenvector equal to $\mathbf{u}_{1}$ corresponding to the Perron root $\widetilde{\sigma}_{1}^{2}$ (left singular vector of $C$ corresponding to the singular value $\widetilde{\sigma}_{1}$ ). Then $\mathbf{u}_{1}$ is an entrywise positive vector and so is the right singular vector $\mathbf{v}_{1}$. Therefore the matrix $\alpha \mathbf{u}_{1} \mathbf{v}_{1}^{T}$ is positive and from Lemma 5 ,

$A=C+\alpha \mathbf{u}_{1} \mathbf{v}_{1}^{T}$ is positive with the prescribed singular values $\sigma_{1}, \sigma_{2}, \ldots, \sigma_{n}$.

Lemma 4 and the form of the matrix in (3.1) allow us to construct a nonnegative matrix , say $C$, whose exponential matrix $e^{C}$ has prescribed singular values: To show this, we consider the matrix $C$ in (3.1). Then a straight forward calculation shows that

$$
C^{m}=\left(\begin{array}{ccccc}
b_{1}^{m} & b_{2} \sum_{k=0}^{m-1} b_{1}^{m-1-k} a_{2}^{k} & \cdots & \cdots & b_{n} \sum_{k=0}^{m-1} b_{1}^{m-1-k} a_{n}^{k} \\
& a_{2}^{m} & & & \\
& & a_{3}^{m} & & \\
& & \ddots & \\
& & & & a_{n}^{m}
\end{array}\right)
$$


and

$$
e^{C}=\sum_{j=0}^{\infty} \frac{1}{j !}\left(\begin{array}{ccccc}
b_{1}^{j} & b_{2} \sum_{k=0}^{j-1} b_{1}^{j-1-k} a_{2}^{k} & \cdots & \cdots & b_{n} \sum_{k=0}^{j-1} b_{1}^{j-1-k} a_{n}^{k} \\
& a_{2}^{j} & & & \\
& & a_{3}^{j} & & \\
& & & \ddots & \\
& & & & a_{n}^{j}
\end{array}\right)
$$

$$
=\left(\begin{array}{ccccc}
\sum_{j=0}^{\infty} \frac{1}{j !} b_{1}^{j} & b_{2} \sum_{j=0}^{\infty} \frac{1}{j !} \sum_{k=0}^{j-1} b_{1}^{j-1-k} a_{2}^{k} & \cdots & \cdots & b_{n} \sum_{j=0}^{\infty} \frac{1}{j !} \sum_{k=0}^{j-1} b_{1}^{j-1-k} a_{n}^{k} \\
\sum_{j=0}^{\infty} \frac{1}{j !} a_{2}^{j} & & & \\
& \ddots & & \\
& & \ddots & \\
& & & \sum_{j=0}^{\infty} \frac{1}{j !} a_{n}^{j}
\end{array}\right)
$$

is also a semibordered diagonal matrix. Now, for $i=2,3, \ldots, n$, we have

$$
\begin{aligned}
\left(e^{C}\right)_{1 i} & =b_{i} \sum_{j=0}^{\infty}\left(\frac{1}{j !} \sum_{k=0}^{j-1} b_{1}^{j-1-k} a_{i}^{k}\right) \\
& =b_{i} \sum_{j=0}^{\infty}\left(\frac{1}{j !} \sum_{k=0}^{j-1} b_{1}^{j-1}\left(\frac{a_{i}}{b_{1}}\right)^{k}\right) \\
& =b_{i} \sum_{j=0}^{\infty}\left(\frac{b_{1}^{j-1}}{j !} \sum_{k=0}^{j-1}\left(\frac{a_{i}}{b_{1}}\right)^{k}\right) .
\end{aligned}
$$

Since

$$
\sum_{k=0}^{j-1}\left(\frac{a_{i}}{b_{1}}\right)^{k}=\frac{1-\left(\frac{a_{i}}{b_{1}}\right)^{j}}{1-\left(\frac{a_{i}}{b_{1}}\right)}=\frac{b_{1}^{j}-a_{i}^{j}}{b_{1}^{j-1}\left(b_{1}-a_{i}\right)}
$$

then 


$$
\begin{aligned}
\left(e^{C}\right)_{1 i} & =\frac{b_{i}}{\left(b_{1}-a_{i}\right)} \sum_{j=0}^{\infty}\left(\frac{b_{1}^{j}-a_{i}^{j}}{j !}\right)=\frac{b_{i}}{\left(b_{1}-a_{i}\right)} \sum_{j=0}^{\infty}\left(\frac{b_{1}^{j}}{j !}-\frac{a_{i}^{j}}{j !}\right) \\
& =\frac{b_{i}\left(e^{b_{1}}-e^{a_{i}}\right)}{b_{1}-a_{i}}, i=2, . ., n .
\end{aligned}
$$

Moreover,

$$
\left(e^{C}\right)_{11}=e^{b_{1}} \text { and }\left(e^{C}\right)_{i i}=e^{a_{i}}, i=2, \ldots, n \text {. }
$$

Hence, given the real positive numbers $\sigma_{1}>\sigma_{2} \geq \cdots \geq \sigma_{n}>0$, from Lemma 4, we may construct a matrix $\widetilde{C}$ of the form (3.1), with singular values $\sigma_{1}, \ldots, \sigma_{n}$, where the $\widetilde{b}_{i}$ are given by (1.3) and (1.4) and can be chosen as positive. Clearly, the $\widetilde{a}_{i}$ can also be chosen positive.

Then, if $e^{C}=\widetilde{C}$, we have

$$
\left(e^{C}\right)_{1 i}=\frac{b_{i}\left(e^{b_{1}}-e^{a_{i}}\right)}{b_{1}-a_{i}}=\widetilde{b}_{i}, i=2, . ., n
$$

and

$$
b_{i}=\frac{\left(b_{1}-a_{i}\right)}{\left(e^{b_{1}}-e^{a_{i}}\right)} \widetilde{b}_{i} \quad i=2, \ldots, n
$$

In the same way,

$$
\left(e^{C}\right)_{11}=e^{b_{1}}=\widetilde{b}_{1}
$$

and

$$
b_{1}=\frac{1}{2} \ln \widetilde{b}_{1}
$$

From (1.3) we see that

$$
\sum_{i=2}^{n}\left(\frac{\widetilde{b}_{i}^{2}}{\widetilde{a}_{i}^{2}-\sigma_{1}^{2}}\right)>0
$$

Finally, from

$$
\left(e^{C}\right)_{i i}=e^{a_{i}}=\widetilde{a}_{i}=\sqrt{\frac{\sigma_{i-1}+\sigma_{i}}{2}}, i=2, \ldots, n,
$$

we have 


$$
a_{i}=\frac{1}{2} \ln \left(\frac{\sigma_{i-1}+\sigma_{i}}{2}\right), i=2, \ldots, n
$$

Observe that if $\sigma_{n}>1$, then $\frac{\sigma_{i}+\sigma_{i-1}}{2}>1$ and the $a_{i}$ are all positive. Thus, if $\sigma_{1}>\sigma_{2} \geq \cdots \geq \sigma_{n}>1$, we can construct a nonnegative matrix $C$ of the form (3.1), in such a way that $e^{C}$ has prescribed singular values $\sigma_{1}, \sigma_{2}, \ldots, \sigma_{n}$. To illustrate this construction, let us consider the numbers $\sigma_{i}: 7,5,3,2$. Then, from (3.2), (3.3) and (3.4) we compute the matrix $C$ such that

$$
e^{C}=\left[\begin{array}{cccc}
3.2845 & 2.428 & 2.7309 & 1.5365 \\
0 & 6.083 & 0 & 0 \\
0 & 0 & 4.1231 & 0 \\
0 & 0 & 0 & 2.5495
\end{array}\right]
$$

has the prescribed singular values $7,5,3,2$.

\section{Positive matrices with prescribed singular values III}

In this section we consider the construction of a rectangular positive matrix with prescribed singular values. First we construct an $m \times n$ nonnegative matrix $B, m>n$, of the form

$$
B=\left(\begin{array}{cccc}
b_{1} & b_{2} & \cdots & b_{n} \\
& a_{2} & & \\
& & \ddots & \\
& & & a_{n} \\
a_{n+1} & & & \\
\vdots & & & \\
a_{m} & & &
\end{array}\right), m>n
$$

and then we apply the perturbation result given by Lemma 5 to fill out $B$ with positive entries.

Theorem 3. Given the numbers $\sigma_{1}>\sigma_{2}>\cdots>\sigma_{n}>0$, there exists an $m \times n$ nonnegative matrix, $m>n$, with singular values $\sigma_{1}, \sigma_{2}, \ldots, \sigma_{n}$. 
Proof. We define

$$
a_{i}=\frac{\sigma_{i-1}+\sigma_{i}}{2}, \quad i=2, \ldots, n,
$$

and we choose $a_{n+1}, a_{n+2} \ldots, a_{m}$ in such a way that

$$
a_{1}^{2}=\sum_{n+1}^{m} a_{j}^{2}<\sigma_{n} .
$$

Since we want to construct the matrix $B$ in (4.1), then we observe that

$$
\mathrm{B}^{T} B=\left(\begin{array}{ccccc}
b_{1}^{2}+\sum_{j=n+1}^{m} a_{j}^{2} & b_{1} b_{2} & b_{1} b_{3} & \cdots & b_{1} b_{n} \\
b_{1} b_{2} & b_{2}^{2}+a_{2}^{2} & b_{2} b_{3} & \ddots & b_{2} b_{n} \\
b_{1} b_{3} & b_{2} b_{3} & \ddots & \ddots & \ddots \\
\vdots & \vdots & \ddots & \ddots & \ddots \\
b_{1} b_{n} & b_{2} b_{n} & \cdots & b_{n-1} b_{n} & b_{n}^{2}+a_{n}^{2}
\end{array}\right)=D^{2}+\mathbf{b b}^{T},
$$

where $D=\operatorname{diag}\left\{a_{1}, \ldots, a_{n}\right\}$ and $\mathbf{b}=\left(b_{1}, \ldots, b_{n}\right)^{T}$. Then

$$
\begin{aligned}
\prod_{k=1}^{n}\left(\sigma_{k}^{2}-\sigma\right) & =\operatorname{det}\left(B^{T} B-\sigma I\right)=\operatorname{det}\left(D^{2}+\mathbf{b b}^{T}-\sigma I\right) \\
& =\operatorname{det}\left(D^{2}-\sigma I\right) \operatorname{det}\left(I+\left(D^{2}-\sigma I\right)^{-1} \mathbf{b} \mathbf{b}^{T}\right)
\end{aligned}
$$

and from the well known formula $\operatorname{det}\left(I+\mathbf{x y}^{T}\right)=1+\mathbf{y}^{T} \mathbf{x}$, we have

$$
\begin{aligned}
\prod_{k=1}^{n}\left(\sigma_{k}^{2}-\sigma\right)= & \left(\sum_{j=n+1}^{m} a_{j}^{2}-\sigma\right) \prod_{k=2}^{n}\left(a_{k}^{2}-\sigma\right)\left(1+\frac{b_{1}^{2}}{\sum_{j=n+1}^{m} a_{j}^{2}-\sigma}+\sum_{k=2}^{n} \frac{b_{k}^{2}}{a_{k}^{2}-\sigma}\right) \\
= & \left(a_{1}^{2}-\sigma\right) \prod_{k=2}^{n}\left(a_{k}^{2}-\sigma\right)+b_{1}^{2} \prod_{k=2}^{n}\left(a_{k}^{2}-\sigma\right)+ \\
& +\left(a_{1}^{2}-\sigma\right) \prod_{k=2}^{n}\left(a_{k}^{2}-\sigma\right) \sum_{k=2}^{n} \frac{b_{k}^{2}}{a_{k}^{2}-\sigma} \\
= & \left(a_{1}^{2}-\sigma\right) \prod_{k=2}^{n}\left(a_{k}^{2}-\sigma\right)+b_{1}^{2} \prod_{\substack{k=2 \\
n}}^{n}\left(a_{k}^{2}-\sigma\right)+ \\
& +\left(a_{1}^{2}-\sigma\right) \prod_{k=2}^{n}\left(a_{k}^{2}-\sigma\right) \sum_{\substack{k=2 \\
k \neq i}}^{n} \frac{b_{k}^{2}}{a_{k}^{2}-\sigma}+b_{i}^{2} \prod_{\substack{k=1 \\
k \neq i}}^{n}\left(a_{k}^{2}-\sigma\right) .
\end{aligned}
$$


Thus, for $\sigma=a_{i}^{2}, i=1, \ldots, n$, we have

$$
b_{i}^{2}=\frac{\prod_{\substack{k=1 \\ k=1 \\ k \neq i}}^{n}\left(\sigma_{k}^{2}-a_{i}^{2}\right)}{\prod_{\substack{k \\ k}}^{n}\left(a_{i}^{2}\right)}, i=1, \cdots n
$$

Since $\sigma_{1}>a_{2}>\sigma_{2}>a_{3}>\cdots>\sigma_{n}>a_{1}$, then the right side in (4.2) is positive and we may choose $b_{i}, i=1, \ldots, n$, as positive. Hence, we may construct the nonnegative matrix $B$ in (4.1), with the prescribed singular values $\sigma_{1}, \sigma_{2}, \ldots, \sigma_{n}$.

Corollary 1. Given the positive numbers $\sigma_{1}>\sigma_{2}>\cdots>\sigma_{n}>0$, there exists an $m \times n$ positive matrix $A, m>n$, with singular values $\sigma_{1}, \sigma_{2}, \ldots, \sigma_{n}$.

Proof. Let $\alpha>0$ such that $\widetilde{\sigma}_{1}=\sigma_{1}-\alpha>\sigma_{2}$. Then from Theorem ?? there exists an $m \times n$ nonnegative matrix $B$ of the form (4.1) with singular values $\widetilde{\sigma}_{1}, \sigma_{2}, . ., \sigma_{n}$. The matrix $B B^{T}$ is irreducible nonnegative and therefore its Perron eigenvector $\mathbf{u}_{1}$ (left singular vector of $B$ corresponding to $\left.\widetilde{\sigma}_{1}\right)$ is positive and so is the right singular vector $\mathbf{v}_{1}$. Hence, from Lemma $5, A=B+\alpha u_{1} v_{1}^{T}$ is an $m \times n$ positive matrix with singular values $\sigma_{1}, \sigma_{2}, \ldots, \sigma_{n}$.

\section{Examples}

Example 1. This example illustrates the construction of the matrix $A$ of Theorem 1: Let $S=\{24,16,16,16,16,2\}$ be given. Since $\epsilon=\frac{\sigma_{1}-\sigma_{2}}{2}=4$, then $\epsilon_{i}=2, i=1,2$. Thus, instead $S$ we consider $\Gamma=\{20,18,18,16,16,2\}$ as the prescribed set of singular values. Then

$$
B_{1}=\left(\begin{array}{cc}
11 & 9 \\
9 & 11
\end{array}\right), \quad B_{2}=\left(\begin{array}{cc}
17 & 1 \\
1 & 17
\end{array}\right), \quad B_{3}=\left(\begin{array}{cc}
17 & 1 \\
1 & 17
\end{array}\right) \text {. }
$$

We apply Lemma 2 to the matrices $B_{1}$ and $B_{2}$, with $\rho=2 \sqrt{2}, \quad \mathbf{u}=\mathbf{v}=$ $\left(\frac{1}{\sqrt{2}}, \frac{1}{\sqrt{2}}\right)^{T}$ to obtain 


$$
\mathcal{B}_{1}=\left(\begin{array}{cc}
B_{1} & \rho \mathbf{u v}^{T} \\
\rho \mathbf{v u} & B_{2}^{T}
\end{array}\right)=\left(\begin{array}{cccc}
11 & 9 & \sqrt{2} & \sqrt{2} \\
9 & 11 & \sqrt{2} & \sqrt{2} \\
\sqrt{2} & \sqrt{2} & 17 & 1 \\
\sqrt{2} & \sqrt{2} & 1 & 17
\end{array}\right),
$$

with eigenvalues $22,16,16,2$. Next we again apply Lemma 2 to the matrices $\mathcal{B}_{1}$ and $B_{3}$, with

$$
\rho=2 \sqrt{3}, \quad \widehat{\mathbf{u}}=\left(\frac{1}{3} \sqrt{3}, \frac{1}{3} \sqrt{3}, \frac{1}{6} \sqrt{6}, \frac{1}{6} \sqrt{6}\right)^{T}, \quad \mathbf{v}=\left(\frac{1}{\sqrt{2}}, \frac{1}{\sqrt{2}}\right)^{T},
$$

to obtain the positive matrix

$$
A=\mathcal{B}_{2}=\left(\begin{array}{cc}
\mathcal{B}_{1} & \rho \widehat{\mathbf{u}} \mathbf{v}^{T} \\
\rho \mathbf{v} \widehat{\mathbf{u}}^{T} & B_{3}
\end{array}\right)=\left(\begin{array}{cccccc}
11 & 9 & \sqrt{2} & \sqrt{2} & \sqrt{2} & \sqrt{2} \\
9 & 11 & \sqrt{2} & \sqrt{2} & \sqrt{2} & \sqrt{2} \\
\sqrt{2} & \sqrt{2} & 17 & 1 & 1 & 1 \\
\sqrt{2} & \sqrt{2} & 1 & 17 & 1 & 1 \\
\sqrt{2} & \sqrt{2} & 1 & 1 & 17 & 1 \\
\sqrt{2} & \sqrt{2} & 1 & 1 & 1 & 17
\end{array}\right)
$$

with the prescribed singular values: $24,16,16,16,16,2$.

Example 2. This example illustrates the construction of a $4 \times 4$ positive nonsymmetric matrix with the prescribed singular values $3, \sqrt{5}, \sqrt{3}, 1$. We consider the list $\sqrt{7}, \sqrt{5}, \sqrt{3}, 1$. Then from Lemma 4 we construct the nonnegative matrix

$$
C=\left(\begin{array}{cccc}
\frac{\sqrt{35}}{4} & \frac{\sqrt{5}}{4} & \frac{3}{4} & \frac{\sqrt{15}}{4} \\
0 & \sqrt{6} & 0 & 0 \\
0 & 0 & 2 & 0 \\
0 & 0 & 0 & \sqrt{2}
\end{array}\right)
$$

with singular values $\sqrt{7}, \sqrt{5}, \sqrt{3}, 1$. Next, we apply Lemma 5 to obtain the positive matrix

$$
\begin{aligned}
A & =C+(3-\sqrt{7}) \mathbf{u}_{1} \mathbf{v}_{1}^{T} \\
& =\left(\begin{array}{cccc}
1.5409 & 0.72275 & 0.82322 & 1.0250 \\
8.4740 \times 10^{-2} & 2.6737 & 0.10026 & 7.7665 \times 10^{-2} \\
3.0943 \times 10^{-2} & 8.1867 \times 10^{-2} & 2.0366 & 2.8359 \times 10^{-2} \\
1.6948 \times 10^{-2} & 4.4837 \times 10^{-2} & 2.0052 \times 10^{-2} & 1.4297
\end{array}\right)
\end{aligned}
$$

with the prescribed singular values $3, \sqrt{5}, \sqrt{3}, 1$. 
Example 3. This example illustrates the construction of a $7 \times 4$ positive matrix with the prescribed singular values $3, \sqrt{5}, \sqrt{3}, 1$. Let $\alpha=3-\sqrt{7}$ and let us consider the numbers $\sqrt{7}, \sqrt{5}, \sqrt{3}, 1$, as in the previous example. From Theorem ??, we compute the nonnegative matrix

$$
B=\left(\begin{array}{cccc}
0.54623 & 0.59252 & 0.84192 & 1.4016 \\
0 & 2.4409 & 0 & 0 \\
0 & 0 & 1.9841 & 0 \\
0 & 0 & 0 & 1.366 \\
0.70711 & 0 & 0 & 0 \\
0.5 & 0 & 0 & 0 \\
0.40825 & 0 & 0 & 0
\end{array}\right)
$$

with singular values $\sqrt{7}, \sqrt{5}, \sqrt{3}, 1$. Since $B^{T} B$ is irreducible nonnegative, its Perron eigenvector, $\mathbf{u}_{1}$, corresponding to the Perron root 7 (left singular vector of $B$ corresponding to the singular value $\sqrt{7}$ ), is entrywise positive and so is the right singular vector $\mathbf{v}_{1}$. Therefore the matrix $\alpha \mathbf{u}_{1} \mathbf{v}_{1}^{T}$ is positive and from Lemma $5, A=B+\alpha \mathbf{u}_{1} \mathbf{v}_{1}^{T}$ is positive with the prescribed singular values $3, \sqrt{5}, \sqrt{3}, 1$.

\section{References}

[1] A. Brauer, Limits for the characteristic roots of a matrix IV: Applications to stochastic matrices, Duke Math. J. 19, pp. 75-91, (1952).

[2] M. Fiedler, Eigenvalues of nonnegative symmetric matrices, Linear Algebra Appl. 9, pp. 119-142, (1974).

[3] R. A. Horn, C..R. Johnson, Topics in Matrix Analysis, Cambridge University Press, Cambridge , (1991).

[4] E. Montaño, M. Salas, R. L. Soto, Nonnegative matrices with prescribed extremal singular values, Computer and Mathematics with Applications, doi:10.1016/j.camwa.2007.11.030, (2007).

\section{Emedin Montaño}

Departamento de Matemática y Física

Universidad de Magallanes

e-mail : emedin.montano@umag.cl 


\section{Mario Salas}

Departamento de Matemáticas

Universidad Católica del Norte

Casilla 1280, Antofagasta

Chile

e-mail : msalas@ucn.cl

and

\section{Ricardo L. Soto}

Departamento de Matemáticas

Universidad Católica del Norte

Casilla 1280, Antofagasta

Chile

e-mail : rsoto@ucn.cl 